\title{
Nanometric Vibration Sensing using Spectral Processing of Laser Self-Mixing Feedback Phase
}

\author{
Nauman Ali, Usman Zabit, Senior Member, IEEE, and Olivier D. Bernal, Member, IEEE \\ DOI:10.1109/JSEN.2021.3083643
}

\begin{abstract}
In this paper, a method is proposed to recover displacements with nanometric precision based on spectral analysis of the feedback phase of self-mixing laser interferometric signal. This method is an enhancement of the previously developed method called Time-domain Frequency-domain Signal Processing (TFSP) which could only recover target vibration(s) with greater than lambda/8 amplitude (where lambda is the laser's wavelength) so long as at least one of the vibration's components has an amplitude greater than or equal to lambda/2. Using TFSP reconstruction as a first approximation of the main target displacement combined with parameter (optical feedback factor C and linewidth enhancement factor alpha) estimation, the proposed method can provide a better in-depth analysis of the self-mixing signal spectrum and thus achieve the detection of even lower amplitude vibrations. Studies presented in the paper indicate that the proposed method does not require accurate parameter estimation. Experimental results obtained using a laser diode based self-mixing sensor with lambda $=\mathbf{7 8 5} \mathbf{n m}$ show that the adding this method on top of TFSP allows to recover target vibrations with amplitude down to approximately lambda/32 with an average amplitude root-mean-square error of $1.56 \mathrm{~nm}$, which corresponds to a 4 fold improvement over TFSP.
\end{abstract}

Index Terms-Vibration measurement, nanometric sensing, self-mixing, optical feedback interferometry, phase unwrapping, spectral analysis.

\section{INTRODUCTION}

$\mathbf{S}$ ELF-MIXING (SM) or optical feedback interferometry (OFI) is an attractive sensing scheme due to the simple, auto-aligned and low-cost nature of laser diode (LD) based sensor [1], [2]. Using this simple opto-electronic system, multiple metric applications have been developed [3]-[6].

Even though resolution of half-wavelength can be easily achieved under moderate optical feedback regime [7], [8] by using the simple SM sensor design shown in Fig. 1, higher resolution can only be reached at the cost of more advanced signal processing techniques. The inherent simplicity of the SM sensor is actually counterbalanced by the complex nature of the SM signal (which is a function of amount of optical feedback). As a result, for open-loop SM signal configuration, recovery of displacement with high accuracy either requires elaborate signal processing [9]-[17] which can be based on phase unwrapping techniques for instance. In addition, using either laser wavelength- [18] or mechanical-modulation [19], open-loop SM signal configurations can also achieve threedimensional imaging of motionless objects by directly exploit-

Nauman Ali and Usman Zabit are with the Department of Electrical Engineering, National University of Sciences and Technology (NUST), Islamabad 44000, Pakistan. (e-mail: usman.zabit@seecs.nust.edu.pk).

Olivier D. Bernal is with LAAS-CNRS, CNRS, University of Toulouse, Institut National Polytechnique Toulouse, 31400 Toulouse, France ing the interferometric phase of SM signal. To alleviate the required amount of signal processing, fringe-locking methods [20]-[22] based on closed-loop configuration have also been successfully developed at the expense of the achievable dynamic range (limited by the laser wavelength tunability) and of a resolution related to the target-laser distance. More recently, instead of exploiting the SM amplitude modulation (AM) channel, narrow band optical frequency filters such as gas cell [23] or Mach-Zehnder [24] have also been proposed to convert the SM frequency modulation channel to detectable AM via photodiode. These methods allow to drastically improve noise performances by at least two decades but required more complex optical processing which might hinder the simplicity of OFI systems.

With the aim to always develop simple yet efficient SM signal processing techniques, we propose here to improve further a recently proposed method called time-domain, frequencydomain processing (TFSP) in [25]. TFSP directly exploits the spectrum of the SM rough phase signal to enable better precision, even under different noise- and optical feedbackconditions [25]. However, TFSP cannot recover nanometric displacements lower than $\lambda / 8$ as it relies on the use of a threshold level in a filtering step to separate the signal (frequency components corresponding to actual target vibrations) from noise and secondary harmonics generated by the rough phase 


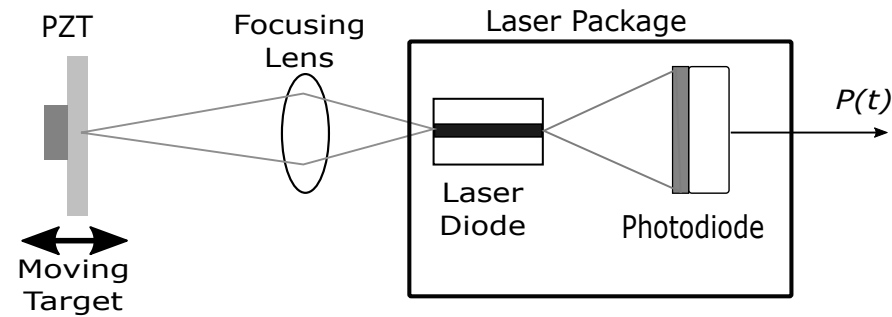

Fig. 1: Block diagram of a typical Self-Mixing (SM) sensor set-up using a laser diode package with its built-in photodiode, and a focusing lens.

estimation of SM signal [25]. Consequently, the proposed approach aims to significantly lower this threshold level. This has been made possible by combining the information related to the major displacements recovered from the TFSP and the estimation of key OFI parameters (optical feedback coupling factor $C$ and the linewidth enhancement factor $\alpha$ ). Note that these estimations need not be very accurate (tolerating $\pm 10 \%$ error), as quantified later in the paper. An expected spectrum of the SM signal corresponding to these main tones can be inferred from this and thus removed from the original SM signal's spectrum. As a result, harmonics corresponding to nanometric displacements can be more easily detected.

The paper is organized as follows. Section - II presents brief theory of OFI while Section-III presents how spectral subtraction is incorporated within TFSP. Detailed simulatedand experimental-results are presented in next two sections, followed by Conclusion.

\section{THEORY OF SELF-MIXING}

Theory of SM is well-established [1], [26], and is briefly summarized below. SM occurs when a portion of the generated laser beam is back-scattered from a target (moving with displacement $D(t)$ ) and re-enters the active laser cavity. This causes a mixing of generated and phase-shifted back-scattered beams. This "self-mixing" causes variation in the optical output power of the laser, denoted as $P(t)$, given by [2]

$$
P(t)=P_{0}\left(1+m \cos \left(\phi_{F}(t)\right)\right)
$$

where $P_{0}$ is the emitted optical power without optical feedback, $m$ is the modulation index and $\phi_{F}(t)$ is the laser output phase in the presence of feedback. $\phi_{F}(t)$ is related to the laser output phase without feedback $\phi_{0}(t)=4 \pi D(t) / \lambda$ by

$$
\phi_{0}(t)=\phi_{F}(t)+C \sin \left(\phi_{F}(t)+\arctan (\alpha)\right)
$$

Depending on $C$, the laser can operate into five different regimes $[1,2]$. SM sensing is generally performed under weak feedback regime $(C<1)$, moderate feedback regime $(1<C<4.6)$, or strong feedback regime $(C>4.6)$. However, moderate feedback regime is usually preferred as the apparently simple saw-tooth shaped SM fringes belonging to such a regime [9] intrinsically provide motion direction indication and do not require advanced SM fringe detection methods e.g. [27].

\section{TFSP AND SPECtRAL SUbTRACTION}

\section{A. TFSP}

As already mentioned, TFSP (see Fig. 2) has advantages over other SM displacement retrieval methods and provides precision of $\lambda / 80$ for micro-metric $(>\lambda)$ harmonic motion [25]. Like other phase unwrapping methods, TFSP requires at least a spectral component of target motion to have an amplitude greater than or equal to $\lambda / 2$ so that at least a complete fringe is generated corresponding to a phase travel of $2 \pi$. However, if a component of target motion has an amplitude less than $\lambda / 8$ then it cannot be recovered by TFSP. This limitation is imposed by the presence of secondary harmonics of rough phase estimation $\hat{\phi}_{F}$ within the spectrum, which have amplitudes well-above the general noise-floor. These secondary harmonics have been simply explained through formation of the Fourier series for the staircase phase signal $\phi_{F s}$, where each step of the staircase phase signal corresponds to individual interferometric fringe corresponding to phaseshift of $2 \pi$ occurring due to target displacement of $\lambda / 2$. This signal mathematically can be expressed as:

$$
\phi_{F s}(t)=2 \pi \sum_{i=1}^{N} \sum_{k=-\infty}^{\infty} \prod\left(\frac{t-(2 k+1) \frac{T}{2}}{T-t_{i}}\right)+\phi_{i n i t}
$$

where $t_{i}$ is the time at which $i^{t h}$ fringe occurs and $N$ is the number of fringes which occur in half of the time period $T$ of target vibration. After some analysis through Fourier series, the amplitude $A_{n}$ of $n^{\text {th }}$ harmonic of $\phi_{F s}$ is given in [25] as,

$$
\forall n \in N^{*} A_{n}=\frac{4}{n} \sum_{i=1}^{N}\left|\sin \left(\frac{2 \pi n}{T} t_{i}\right)\right|
$$

Using this equation, the amplitude of harmonics of original target vibration present in $\phi_{F s}$ can easily be computed. Thus, at a primary level, it can be deduced that almost all the information regarding the target vibration is present in the first harmonic of $\phi_{F s}$. As computed in [25], if only $\phi_{F s}$ spectrum is used then the maximum error which can occur in $A_{1}$ is less than $80 \mathrm{~nm}$ if $A_{p-p}$ of target vibration is of the order of laser wavelength $\lambda$ of $785 \mathrm{~nm}$. However, this error rapidly decreases as the amplitude of target vibration increases. Note that if $\hat{\phi}_{F}$ is used instead of $\phi_{F s}$ then precision performance of TFSP approached $\lambda / 100$ for harmonic micro-metric target vibrations [25]. So, when retrieving the first harmonic, the threshold may be set as minimum as the amplitude of secondary harmonic (which refers to any harmonic with the highest amplitude other than the first harmonic). Based on this simplified theoretical analysis, it was shown in [25] that a conservative value for the threshold $\phi_{p r i}$ is $\pi / 2$ which then corresponds to target vibration of amplitude $\lambda / 8$.

\section{B. Spectral Subtraction}

In the light of above discussion, it is seen that TFSP can not detect vibrations with amplitudes less than $\lambda / 8$. So, in this paper, we propose the use of spectral subtraction to improve TFSP by effectively lowering the threshold (which is used to separate the target vibration components from noise within spectrum of $\hat{\phi}_{F}$ ). 


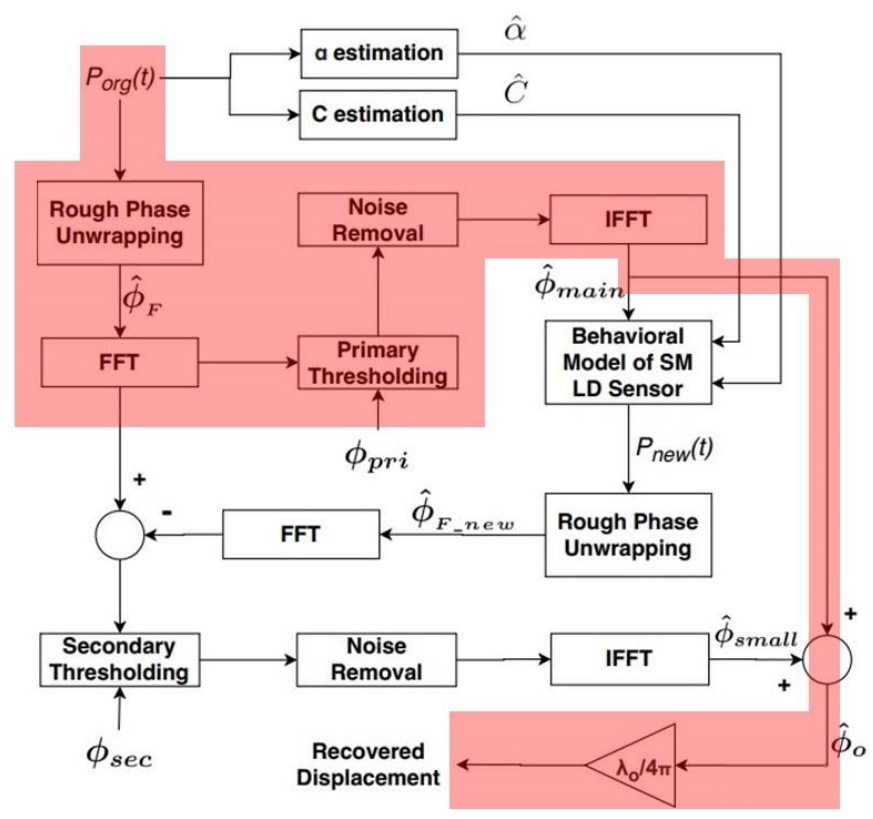

(a)

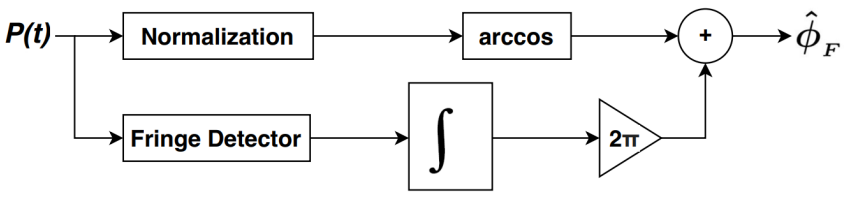

(b)

Fig. 2: Schematic diagram of (a) proposed spectral subtraction based TFSP (SC-TFSP), where original TFSP [25] is shaded in red, and (b) rough phase unwrapping.

As the primary threshold is limited by the peaks occurring at harmonics of the main tone (highest amplitude tone) so reduction in the amplitude of these harmonics enables potential lowering of the primary threshold. However, note that this is not achieved by removing the spectral content at all the harmonics of the main tone (as some other actual target motion component(s) can also exist at one (or more) harmonic multiples of the main tone). Instead, this is achieved by 1) generating the time-domain SM signal $P_{\text {new }}(t)$ (using the SM behavioral model [26]) corresponding to the main tone within recovered $\hat{\phi}_{F}$ 's spectrum, followed by 2) rough unwrapping of $P_{\text {new }}(t)$ resulting in generation of new spectrum $\hat{\phi}_{F_{\text {new }}}$. Subtraction of this new spectrum from $\hat{\phi}_{F}$ results in removal of actual contribution of harmonics of main tone from spectrum of $\hat{\phi}_{F}$. This then significantly lowers the amplitudes of all secondary tones related to the main tone. Consequently, this makes it possible to recover smaller than $\lambda / 8$ amplitude target vibration tone(s) from the modified spectrum by using a new and lower threshold. Such an approach permits to process target motion which may be composed of multiple highamplitude tones as well as small-amplitude tones which may occur at harmonic multiples of one another.

In order to implement this spectral subtraction, signal processing within TFSP needs to be significantly changed (see Fig 2(a)b. The first step of TFSP is used to recover the main-tone $\hat{\phi}_{\text {main }}$ from $\hat{\phi}_{F}$ corresponding to the original SM signal $P_{\text {org }}(t)$ by using a primary threshold $\phi_{p r i}$. Then, the corresponding $\hat{\phi}_{\text {main }}$ is used to generate the equivalent SM signal $P_{\text {new }}(t)$ (see Fig. 3(a) by using the SM behavioural model developed in [26]. This model requires three inputs: $\phi_{0}(t)=4 \pi D(t) / \lambda_{o}, C$ factor, and $\alpha$ factor to provide the output denoted $P_{\text {new }}(t)$.

As $\hat{\phi}_{0}(t)$ is already recovered in TFSP so the $C$ factor and the $\alpha$ factor corresponding to original $P_{\text {org }}(t)$ need to be estimated. As discussed in the next section, it turns out that these estimations need not to be very accurate. Thus, simple $C$ estimation $\hat{C}$ [12] and $\alpha$ estimation $\hat{\alpha}$ [28] methods have been employed in the presented work.

The second step is to roughly unwrap the phase of the generated SM signal $P_{\text {new }}(t)$ which is performed along the same lines as in TFSP, as shown in Fig. 2(b) Thus, the new feedback phase $\hat{\phi}_{F-n e w}$ is obtained. Then its Fast Fourier Transform (FFT) is computed to obtain its spectrum which is then subtracted from the spectrum of the originally estimated feedback phase $\hat{\phi}_{F}$ by using $P_{\text {org }}(t)$, as shown in Fig. 2(a)

After the subtraction, it can be observed in the modified spectrum (shown in Fig. 3 (c), Fig. 10 (b), and Fig. 11 (b) for their respective cases) that the amplitudes of harmonics of main tone have been significantly reduced in the modified spectrum such that the small tone(s) which was(were) hidden in the spectrum of $\hat{\phi}_{F}$ can now be seen as the highest amplitude component(s). Thus, one can easily retrieve such target vibration component(s) with amplitude less than $\lambda / 8$ and remove all the noise left in the spectrum. Use of Inverse Fast Fourier Transform (IFFT) eventually recovers the small tone in time-domain (see Fig. 4(b)]. Finally, addition of maintone(s) with small-tone(s) completes the recovery of target motion (see Fig. 4(a) . This proposed algorithm is called spectral cleaning based TFSP, and is denoted as SC-TFSP.

Furthermore, it may be noted that SC-TFSP, in a manner similar to TFSP and other phase unwrapping methods, requires the presence of at least a target motion component (denoted as the main tone in the previous discussion) with amplitude greater than $\lambda / 2$. In case an actual nano-metric remote target motion is devoid of any such component then SC-TFSP can still be used by generating such a motion component (which is later removed) either by vibrating the laser stage or by modulating the laser current, as demonstrated in [6].

\section{Secondary Threshold}

Determination of the secondary threshold $\phi_{s e c}$ is an important part of SC-TFSP because it will specify the smallest vibration which can be consistently detected despite variations in different parameters. As the amplitude of SM signal's secondary harmonics depends on 1) the sampling frequency $\left.F_{s}, 2\right)$ the amplitude of main tone, 3) $C$, 4) error in $\left.\hat{C}, 5\right) \alpha$, and 6) error in $\hat{\alpha}$, therefore many simulations were performed as a function of these parameters to find an optimal value for $\phi_{s e c}$ under noiseless conditions for a laser with $\lambda=785 \mathrm{~nm}$.

To determine the impact of SM signal's sampling frequency, the simulations were carried out at $F_{s}=1$ MSPS (Fig. 5) and $F_{s}=10$ MSPS (Fig. 6 and Fig. 7). By comparing Fig. 


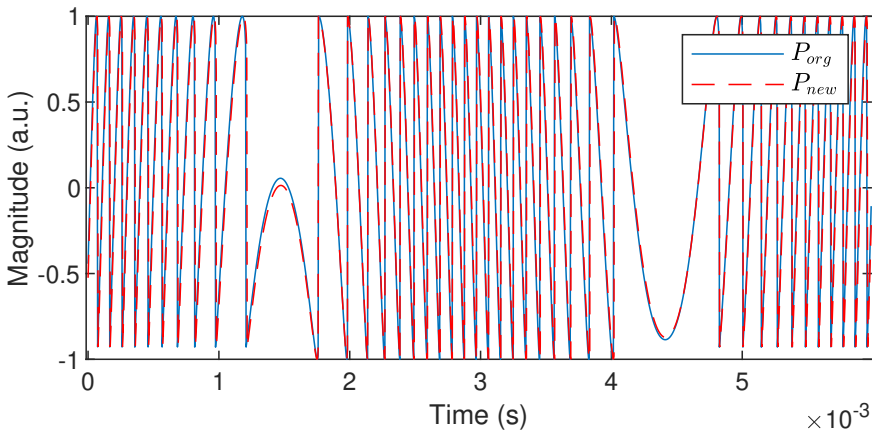

(a) Original SM Signal $P_{\text {org }}(t)$ (blue) and SM signal $P_{\text {new }}(t)$ (red) generated from $\hat{\phi}_{\text {main }}$.

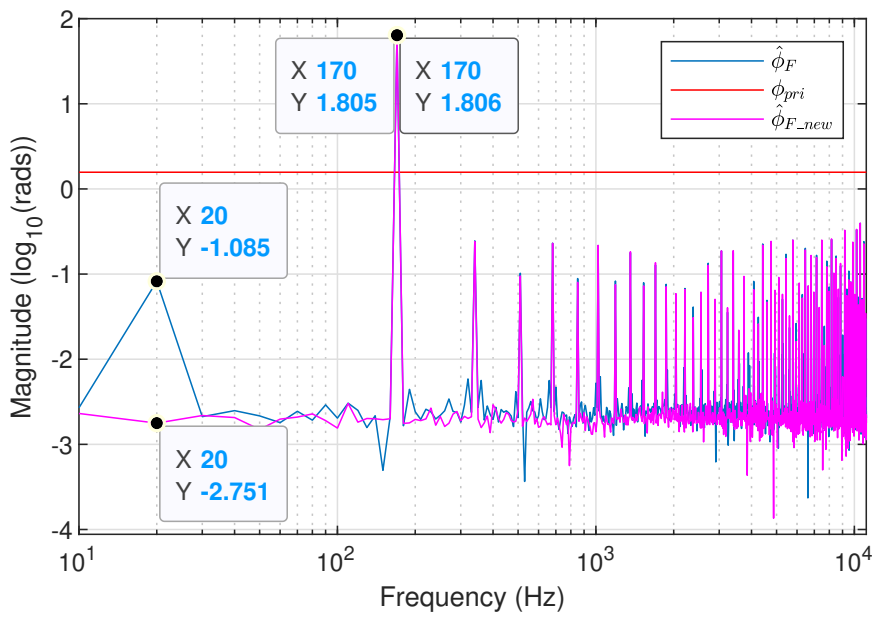

(b) Spectrum of $\hat{\phi}_{F}$ (blue), primary threshold $\phi_{\text {pri }}$ (red) and Spectrum of $\hat{\phi}_{\text {F_new }}$ (magenta).

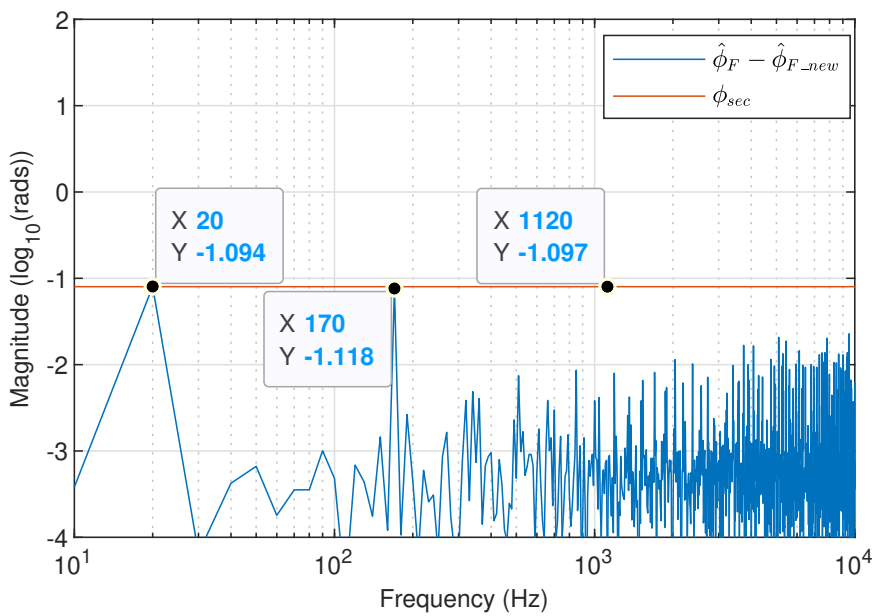

(c) Spectrum after the subtraction (blue) and secondary threshold $\phi_{\sec }($ red)

Fig. 3: Simulated extracted $P_{\text {new }}(t)$ shown in (a) and its corresponding spectrum $\hat{\phi}_{F \_n e w}$ shown in (b) obtained by the spectral cleaning based TFSP (SC-TFSP) from the original SM signal (shown in (a)) generated for a target motion composed of a tone $\phi_{\text {main }}$ of $4 \mu \mathrm{m}$ amplitude at $170 \mathrm{~Hz}$ and another tone $\phi_{\text {small }}$ of $5.5 \mathrm{~nm}$ amplitude at $20 \mathrm{~Hz}$, where $\lambda=785 \mathrm{~nm}$ and spectral subtraction (shown in (c)) of $\hat{\phi}_{F_{-} \text {new }}$ and $\hat{\phi}_{F}$.

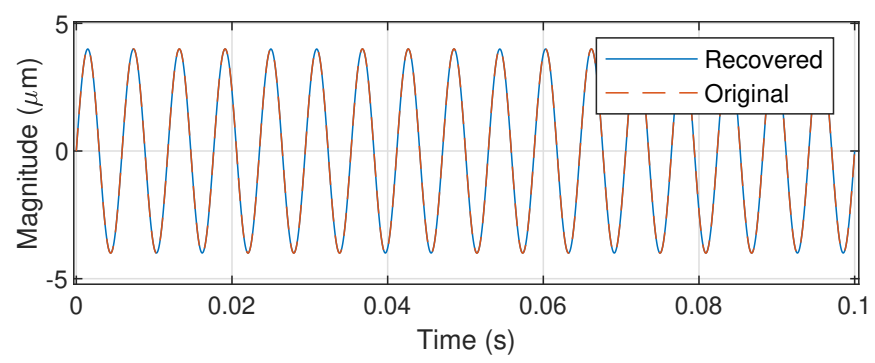

(a) Recovered main tone $\hat{\phi}_{\text {main }}$ (blue) and Original main tone $\phi_{\text {main }}$ (red).

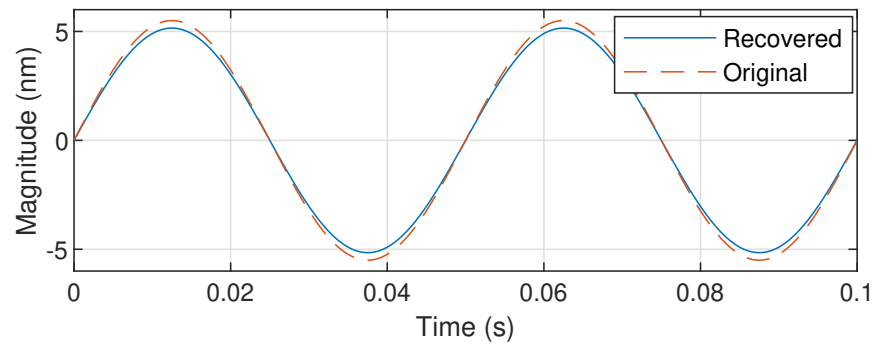

(b) Recovered small tone $\hat{\phi}_{\text {small }}$ (blue) and Original small tone $\phi_{\text {small }}$ (red).

Fig. 4: Reconstructed main- and small-tone displacement by SC-TFSP (shown in (a) and (b), respectively) for a target motion composed of a tone $\phi_{\text {main }}$ of $4 \mu \mathrm{m}$ amplitude at 170 $\mathrm{Hz}$ and another tone $\phi_{\text {small }}$ of $5.5 \mathrm{~nm}$ amplitude at $20 \mathrm{~Hz}$, where $\lambda=785 \mathrm{~nm}$.

5 and Fig. 6, it can be seen that in case the SM signal is not sufficiently highly sampled then incorrect measurement of secondary harmonics can occur. This can be seen in Fig. 5 in which even under assumption of perfect $C$ estimation, secondary harmonic amplitude in the subtracted spectrum initially decreases but then begins to increase with increase in target's main tone vibration amplitude; a trend which is unusual, and doesn't recur at higher $F_{s}$, as seen in Fig. 6 .

As the amplitude of secondary harmonics also depends on $C$, and as SC-TFSP requires $\hat{C}$, so simulations were done to ascertain the amplitude of secondary harmonics before and after the spectral subtraction in case of perfectly estimated $C$ and in cases of $\pm 10 \%$ error in $\hat{C}$ (see Fig. 6 and Fig. 7).

Fig. 6 indicates that in case of high accuracy $\hat{C}$, max. amplitude of secondary harmonics in the subtracted spectrum remains $<0.04$ rads if main-tone amplitude is $>5 \lambda$.

Focusing on Fig. 7 (b and c), it can be seen that despite the increase in the amplitude of secondary harmonics due to increase in $C$, SC-TFSP results in consistently small amplitude of secondary harmonic after spectral subtraction $(\approx 0.04 \mathrm{rads})$ for $1 \leq C \leq 4$, again in case of high accuracy $C$ estimation.

Focusing on Fig. 8 (a), it can be seen that the amplitude of secondary harmonics in the subtracted spectrum is negligibly affected in case of variation in $\alpha$. Likewise, Fig. 8 (b) indicates that even large error in $\hat{\alpha}$ also has a negligible effect.

To conclude, in the absence of additive wide-band noise [29] and speckle [8] that can affect SM signal, these simulations indicate that the value of $\phi_{\text {sec }}$ can be set to the minimum of $0.08 \mathrm{rads}\left(\approx 5.0 \mathrm{~nm}\right.$ by using $\phi_{0}(t)=4 \pi D(t) / \lambda$ and $\lambda=785$ $\mathrm{nm}$ ) in case the amplitude of main tone $>5 \lambda$ and $1 \leq C \leq 4$ 


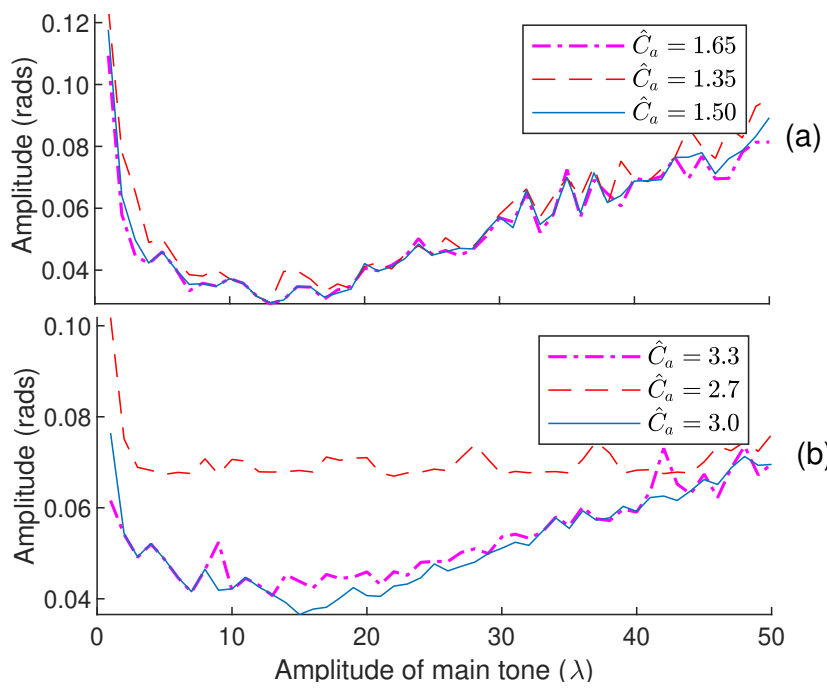

Fig. 5: Simulated result of spectral cleaning based TFSP (SCTFSP) in case of target motion composed of main tone $\phi_{\text {main }}$ at $170 \mathrm{~Hz}$ and another tone of $10 \mathrm{~nm}$ amplitude at $20 \mathrm{~Hz}$ with $\alpha=5$ sampled at 1 MSPS. (a) Amplitude of secondary harmonic in the subtracted spectrum with $\hat{C}$ assigned the value $\hat{C}_{a}$ of 1.65 (Magenta), 1.35 (Red) and 1.5 (blue) vs amplitude of main tone where $\mathrm{C}=1.5$. (b) Amplitude of secondary harmonic in the subtracted spectrum with $\hat{C}$ assigned the value $\hat{C}_{a}$ of 3.3 (Magenta), 2.7 (Red) and 3.0 (blue) vs amplitude of main tone where $C=3.0$.

while tolerating $\pm 10 \%$ error in $\hat{C}$.

\section{Performance of Spectral Cleaning based TFSP}

The performance of proposed SC-TFSP has been verified with respect to various SM- and target motion-based parameters by considering $\lambda=785 \mathrm{~nm}$ in the absence of noise. The $F_{s}$ for SM signal was kept at 1 MSPS and it was generated for the duration of $0.1 \mathrm{~s}$.

\section{A. Performance with respect to $C$ and alpha factors}

Table I presents simulation based RMS error results of SCTFSP as a function of $C$ with $\alpha=5$. Remote motion is composed of a tone of $4 \mu \mathrm{m}$ at $170 \mathrm{~Hz}$ and another tone of $25 \mathrm{~nm}$ at $20 \mathrm{~Hz}$. SC-TFSP based motion retrieval is compared with reference target motion to obtain error performance. These simulations suggest that the recovery of small tone is robust against variations in $C$. Likewise, Table $\Pi$ in indicates that variation in $\alpha$ does not have a significant impact on the performance of the proposed method.

\section{B. Effect of error in C estimation}

The simple method used within SC-TFSP to estimate the value of $C$ of original SM signal has an accuracy of about $10 \%$ [12]. So, simulations were conducted to test the robustness of the proposed method against possibly large errors in $C$ estimation (see Table IIII). For these simulations, original SM signal $P_{\text {org }}(t)$ has $C=1.5$ and 3 while $P_{\text {new }}(t)$ is generated

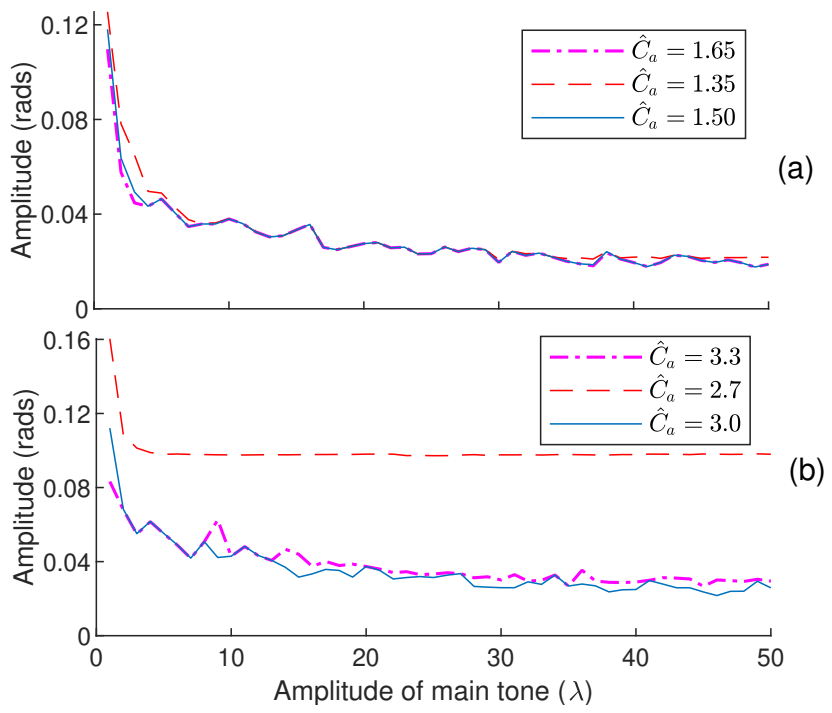

Fig. 6: Effect of increase in $F_{s}$ from 1 MSPS to 10 MSPS on amplitude of secondary harmonic in the subtracted spectrum with $\hat{C}$ assigned the value $\hat{C}_{a}$. All other parameters remain the same as reported in caption of Fig. 5 .

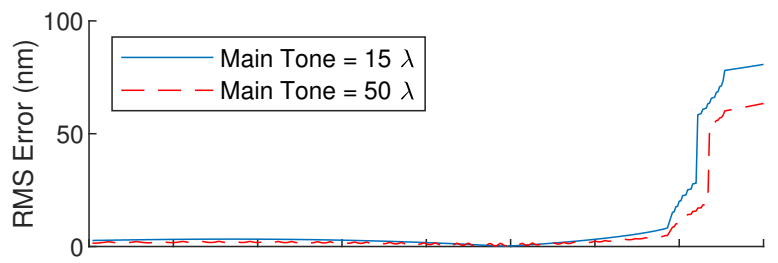

(a)

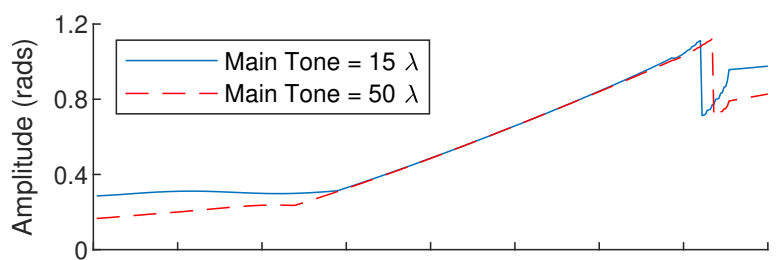

(b)

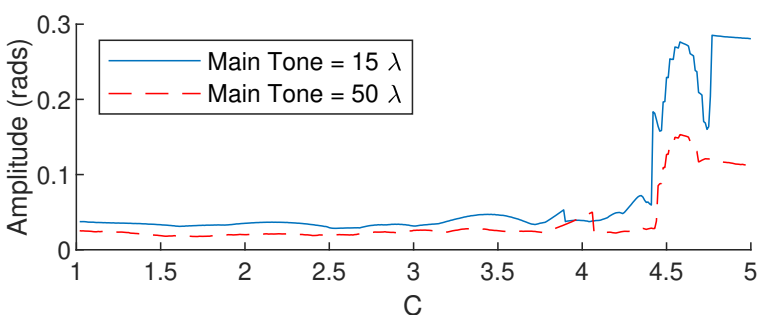

(c)

Fig. 7: Simulated result of SC-TFSP in case of target motion composed of main tone $\phi_{\text {main }}$ of $15 \lambda$ (blue line) and $50 \lambda$ (dashed line in red) amplitudes at $170 \mathrm{~Hz}$ and another tone of $10 \mathrm{~nm}$ amplitude at $20 \mathrm{~Hz}$ with $\alpha=5$ sampled at 10 MSPS. (a) RMS error in main tone vs $C$. (b) Amplitude of secondary harmonic in the spectrum of $\hat{\phi}_{F}$ vs $C$. (c) Amplitude of secondary harmonic in the subtracted spectrum with $\hat{C}$ assigned the value equal to $C$ vs $C$. 

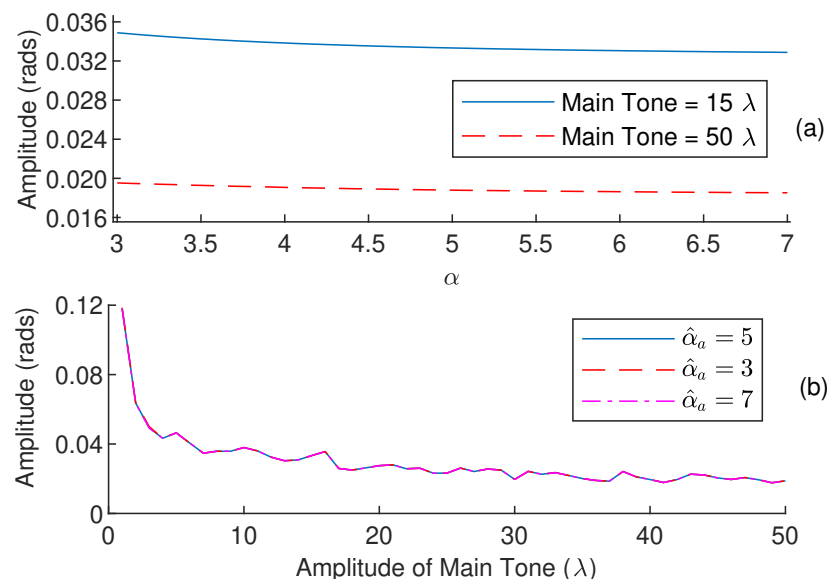

Fig. 8: Simulated result of SC-TFSP in case of target motion composed of main tone $\phi_{\text {main }}$ at $170 \mathrm{~Hz}$ and another tone of $10 \mathrm{~nm}$ amplitude at $20 \mathrm{~Hz}$ with $C=1.5$ sampled at 10 MSPS. (a) Amplitude of secondary harmonic in the subtracted spectrum with $\hat{\alpha}$ assigned the value equal to $\alpha$ vs $\alpha$ for main tone amplitude of $15 \lambda$ (blue line) and $50 \lambda$ (dashed line in red). (b) Amplitude of secondary harmonic in the subtracted spectrum for actual $\alpha=5$ with $\hat{\alpha}$ assigned the value $\hat{\alpha}_{a}$ of 7 (dash-dotted line in magenta), 3 (dashed line in red) and 5 (blue line) vs amplitude of main tone.

TABLE I: Simulation based RMS error results of the proposed SC-TFSP as a function of $C$ with $\alpha=5$ for target motion composed of a tone of $4 \mu \mathrm{m}$ at $170 \mathrm{~Hz}$ and another tone of $25 \mathrm{~nm}$ at $20 \mathrm{~Hz}$, with $F_{s}$ for SM signal of 1 MSPS.

\begin{tabular}{|c|c|c|c|c|}
\hline$C$ & $\hat{C}$ & $\begin{array}{c}\text { Error in Main Tone } \\
(\mathrm{nm})\end{array}$ & $\begin{array}{c}\text { Error in Small Tone } \\
(\mathrm{nm})\end{array}$ & $\begin{array}{c}\text { Total Error } \\
(\mathrm{nm})\end{array}$ \\
\hline 1.5 & 1.500 & 1.8099 & 1.4478 & 2.3177 \\
\hline 2.0 & 2.000 & 2.4219 & 1.6181 & 2.9129 \\
\hline 2.5 & 2.499 & 3.2105 & 1.6632 & 3.6164 \\
\hline 3.0 & 2.999 & 4.6320 & 1.7027 & 4.9365 \\
\hline 3.5 & 3.500 & 6.7625 & 1.6448 & 6.9615 \\
\hline 4.0 & 3.999 & 9.4064 & 1.4718 & 9.5215 \\
\hline
\end{tabular}

with $\hat{C}$ assigned a value $\hat{C}_{a}$ between 1.0 to 4.0 . Result of 'NA' i.e. 'not applicable' indicates that the small tone is not recovered as its amplitude remains below that of other harmonics even after spectral subtraction.

The tabulated results indicate that the recovery of small tone can be compromised in case of large error in $C$ estimation. This is understandable because the amplitude of secondary harmonics depends on $C$ (see Fig. 7 (b) and [25]). So, when $\Delta C=|C-\hat{C}|$ increases, then harmonics of main tone in spectrum of $\hat{\phi}_{F_{-} \text {new }}$ have significantly different amplitude than those in spectrum of $\hat{\phi}_{F}$. Consequently, spectral subtraction does not provide as effective suppression of harmonics' amplitude, thereby making it difficult or even impossible to extract the actual small tone if amplitude of any subtracted harmonic becomes greater than that of small tone. Furthermore, as seen in Table III, impact of inaccurate $C$ estimation is increased in case the small tone lies at a harmonic of main tone, as also highlighted by the study of effect of $C$ on the spectrum of SM signal presented in [6]. Note that results of SC-TFSP in cases where small tone(s) lie at harmonic(s) of main tone are
TABLE II: Simulation based RMS error results of the proposed SC-TFSP as a function of $\alpha$ with $C=1.5$ for target motion composed of a tone of $4 \mu \mathrm{m}$ at $170 \mathrm{~Hz}$ and another tone of $25 \mathrm{~nm}$ at $20 \mathrm{~Hz}$.

\begin{tabular}{|c|c|c|c|c|}
\hline$\alpha$ & $\hat{\alpha}$ & $\begin{array}{c}\text { Error in Main Tone } \\
(\mathrm{nm})\end{array}$ & $\begin{array}{c}\text { Error in Small Tone } \\
(\mathrm{nm})\end{array}$ & $\begin{array}{c}\text { Total Error } \\
(\mathrm{nm})\end{array}$ \\
\hline 3 & 2.91 & 1.8699 & 1.4447 & 2.3630 \\
\hline 4 & 3.65 & 1.8197 & 1.4450 & 2.3237 \\
\hline 5 & 4.36 & 1.8099 & 1.4478 & 2.3177 \\
\hline 6 & 4.97 & 1.8110 & 1.4454 & 2.3172 \\
\hline 7 & 5.88 & 1.8144 & 1.4458 & 2.3200 \\
\hline
\end{tabular}

TABLE III: Simulation based RMS error results of the proposed SC-TFSP as a function of estimated value of feedback coupling factor $\hat{C}$ with $\alpha=5$ for target motion composed of a tone of $4 \mu \mathrm{m}$ of frequency $f_{1}$ and another tone of $25 \mathrm{~nm}$ of frequency $f_{2}$.

\begin{tabular}{|c|c|c|c|c|}
\hline \multirow{3}{*}{$\hat{C}_{a}$} & \multicolumn{2}{|c|}{$f_{1}=30 \mathrm{~Hz} \& f_{2}=90 \mathrm{~Hz}$} & \multicolumn{2}{c|}{$f_{1}=170 \mathrm{~Hz} \& f_{2}=20 \mathrm{~Hz}$} \\
\cline { 2 - 5 } & $C=1.5$ & $C=3.0$ & $C=1.5$ & $C=3.0$ \\
\cline { 2 - 5 } & $\begin{array}{c}\text { Error in } \\
\text { Small Tone } \\
(\mathrm{nm})\end{array}$ & $\begin{array}{c}\text { Error in } \\
\text { Small Tone } \\
(\mathrm{nm})\end{array}$ & $\begin{array}{c}\text { Error in } \\
\text { Small Tone } \\
(\mathrm{nm})\end{array}$ & $\begin{array}{c}\text { Error in } \\
\text { Small Tone } \\
(\mathrm{nm})\end{array}$ \\
\hline 1.0 & 2.1792 & 8.6112 & 1.4463 & NA \\
\hline 1.5 & 2.8825 & 6.4704 & 1.4478 & NA \\
\hline 2.0 & 2.9796 & 3.2729 & 1.4472 & 1.7027 \\
\hline 2.5 & 1.6576 & 0.4156 & 1.4445 & 1.7026 \\
\hline 3.0 & NA & 3.8579 & 1.4462 & 1.7019 \\
\hline 3.5 & NA & NA & 1.4454 & 1.7037 \\
\hline 4.0 & NA & NA & 1.4448 & 1.7033 \\
\hline
\end{tabular}

presented in a later sub-section.

\section{Effect of error in alpha estimation}

In a similar manner, tests were also conducted to quantify the effect of error in $\alpha$ estimation on the error performance of SC-TFSP (see Table IV). For these simulations, $P_{\text {org }}(t)$ has $\alpha=5$ while $P_{\text {new }}(t)$ is generated with $\hat{\alpha}$ assigned a value $\hat{\alpha}_{a}$ between 3.0 and 7.0. Even this large error in $\alpha$ estimation resulted in maximum error of $1 \mathrm{~nm}$ as compared to the performance for $\alpha$ matched $P_{n e w}(t)$.

\section{Effect of variation in $C$ on secondary threshold}

During real-world experimental SMI signal acquisition, it is possible that the optical feedback coupling does not remain constant but varies continuously over time, e.g. due to speckle [8]. So, to determine the effect of continuous variation in $C$ on the ability to recover small tone(s) which in turn depends on $\phi_{\text {sec }}$, simulations were conducted under noiseless conditions

TABLE IV: Simulation based RMS error results of SC-TFSP as a function of $\hat{\alpha}$ with $C=1.5$ and $\alpha=5$ for target motion with a tone of $4 \mu \mathrm{m}$ at $170 \mathrm{~Hz}$ and another tone of $25 \mathrm{~nm}$ at $170 \mathrm{~Hz}$.

\begin{tabular}{|c|c|c|c|}
\hline$\hat{\alpha}_{a}$ & $\begin{array}{c}\text { Error in Main Tone } \\
(\mathrm{nm})\end{array}$ & $\begin{array}{c}\text { Error in Small Tone } \\
(\mathrm{nm})\end{array}$ & $\begin{array}{c}\text { Total Error } \\
(\mathrm{nm})\end{array}$ \\
\hline 3 & 1.8699 & 1.4451 & 2.3633 \\
\hline 4 & 1.8197 & 1.4451 & 2.3238 \\
\hline 6 & 1.8110 & 1.4453 & 2.3171 \\
\hline 7 & 1.8144 & 1.4455 & 2.3198 \\
\hline
\end{tabular}


with $\lambda=785 \mathrm{~nm}, \alpha=5$ while $C$ was varied by $10 \%$ in a sinusoidal manner at the main-tone frequency of $170 \mathrm{~Hz}$ with mean $C$ value of 1.5. This variation in $C$ is shown in Fig. 9 (a). Fig. 9 (b) presents SM signal for main-tone amplitude of 2 $\mu \mathrm{m}$ and continuously varying $C$ shown in Fig. 9 (a). Fig. 9 (c) shows the amplitude of secondary harmonic (which sets the minimum possible value for $\phi_{s e c}$ ) as a function of assigned $C$ value $C_{a}$.

The results indicate that $\phi_{\sec }$ can be assigned the value of 0.32 radians under noiseless conditions. However, to cater to the noise present in experimental SM signals (see next section), $\phi_{\text {sec }}$ was assigned a conservative value of 0.36 radians $(\approx \lambda / 35)$.

\section{E. Multiple Target Vibration Components}

Additional tests were conducted to quantify the performance of SC-TFSP in case more than two frequency components composed the target motion, and also in case if a target frequency component is itself at a harmonic of another target frequency component (see Table Vp. Fig. 10 illustrates the process of recovering multiple small tones for the case of target motion composed of tones of $4 \mu \mathrm{m}$ amplitude at $100 \mathrm{~Hz}, 170$ $\mathrm{Hz}$, and $340 \mathrm{~Hz}$, and tones of $25 \mathrm{~nm}$ amplitude at $200 \mathrm{~Hz}$, $300 \mathrm{~Hz}$, and $400 \mathrm{~Hz}$.
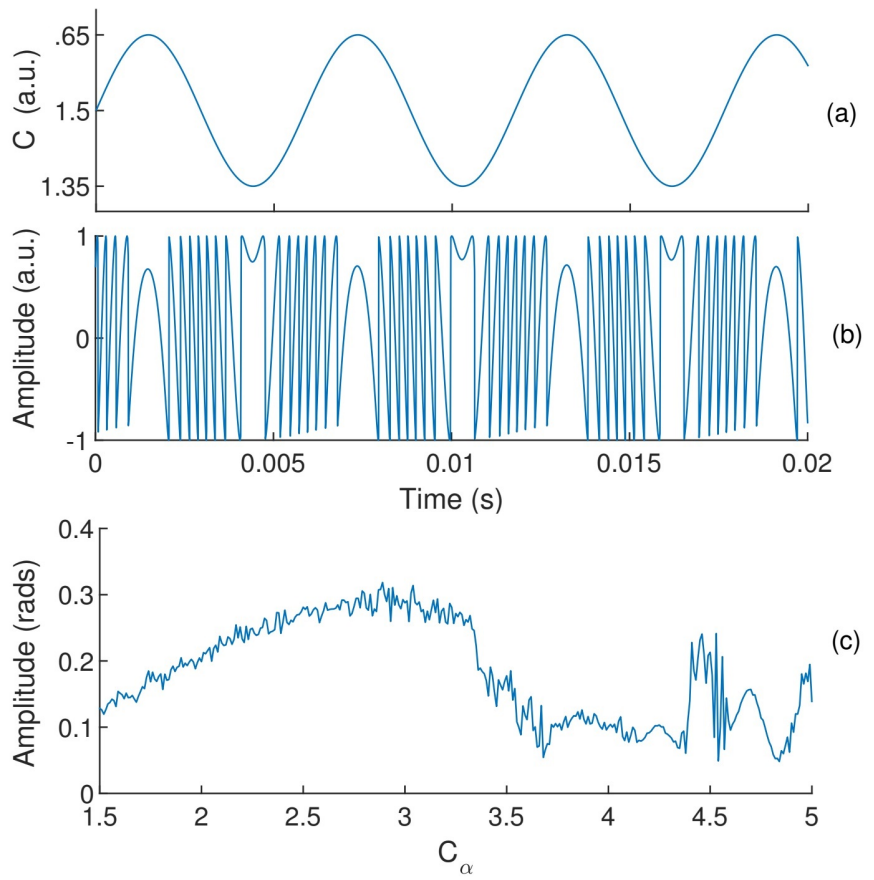

Fig. 9: Simulated result of SC-TFSP in case $C$ varies by $10 \%$ in a sinusoidal manner at the main-tone frequency of $170 \mathrm{~Hz}$ with mean $C$ value of 1.5 while target motion is composed of a main tone $\phi_{\operatorname{main}}$ at $170 \mathrm{~Hz}$ with amplitude of $2 \mu \mathrm{m}$ and another tone of $10 \mathrm{~nm}$ amplitude at $20 \mathrm{~Hz}$ with $\alpha=5$, and $F_{s}=1$ MSPS: (a) Time-variation in $C$, (b) original SM signal $P_{\text {org }}(t)$, and (c) amplitude of secondary harmonic vs $\hat{C}_{a}$.
TABLE V: Simulated RMS error results of SC-TFSP as a function of frequency components in the target motion with $C=1.5$ and $\alpha=5$. The main- and small-tones are of $4 \mu \mathrm{m}$ and $25 \mathrm{~nm}$.

\begin{tabular}{|c|c|c|c|c|c|}
\hline $\begin{array}{c}\text { Main } \\
\text { Tones } \\
(\mathrm{Hz})\end{array}$ & $\begin{array}{c}\text { Small } \\
\text { Tones } \\
(\mathrm{Hz})\end{array}$ & $\hat{C}$ & $\begin{array}{c}\text { Error in } \\
\text { Main } \\
\text { Tones } \\
(\mathrm{nm})\end{array}$ & $\begin{array}{c}\text { Error in } \\
\text { Small } \\
\text { Tones } \\
(\mathrm{nm})\end{array}$ & $\begin{array}{c}\text { Total } \\
\text { Error } \\
(\mathrm{nm})\end{array}$ \\
\hline 170 & 340 & 1.5000 & 1.7741 & 0.0533 & 1.7749 \\
\hline 100,170 & 340 & 1.5034 & 2.4765 & 0.2157 & 2.4859 \\
\hline $100,170,340$ & 300 & 1.5274 & 3.4931 & 0.5015 & 3.5289 \\
\hline $100,170,340$ & 200,300 & 1.5281 & 3.4366 & 0.7103 & 3.5093 \\
\hline $100,170,340$ & $200,300,400$ & 1.5290 & 3.2652 & 1.1007 & 3.4457 \\
\hline $100,500,600$ & $200,300,400$ & 1.5761 & 7.5388 & 1.9717 & 7.7920 \\
\hline
\end{tabular}

TABLE VI: Experimental data-set based performance of SCTFSP for PZT motion with a $170 \mathrm{~Hz}$ tone of $4.4 \mu \mathrm{m}$ and a $20 \mathrm{~Hz}$ tone of $25 \mathrm{~nm}$.

\begin{tabular}{|c|c|c|c|c|}
\hline $\begin{array}{c}\text { Sr. } \\
\text { No. }\end{array}$ & $\begin{array}{c}\text { Main Tone } \\
(\mathrm{um})\end{array}$ & $\begin{array}{c}\text { Small Tone } \\
(\mathrm{nm})\end{array}$ & $\begin{array}{c}\text { Error in small tone } \\
(\mathrm{nm})\end{array}$ & $\hat{C}$ \\
\hline 1 & 4.34 & 24.10 & 0.90 & 2.20 \\
\hline 2 & 4.36 & 22.58 & 2.42 & 2.20 \\
\hline 3 & 4.36 & 22.96 & 2.04 & 2.19 \\
\hline 4 & 4.37 & 23.81 & 1.19 & 2.15 \\
\hline 5 & 4.37 & 23.74 & 1.26 & 2.15 \\
\hline Avg. & 4.36 & 23.44 & 1.56 & 2.18 \\
\hline
\end{tabular}

\section{EXPERIMENTAL RESULTS}

To validate the proposed method, we set-up an experiment with an SM sensor based on Hitachi HL7851 laser diode package. The emitted wavelength was $785 \mathrm{~nm}$ with output power of $50 \mathrm{~mW}$. The SM signal was acquired by using the built-in photo diode, located at the back-facet of the LD. A commercial piezoelectric transducer from Physik Instrumente (P753.2CD) has been used as target. It has a capacitive feedback sensor with $2 \mathrm{~nm}$ resolution which was later used as reference sensor to quantify error performance.

As stated previously, secondary threshold $\phi_{s e c}$ for experimental SM signals is set to 0.36 radians (corresponding to 4.5 times the simulated threshold) in order to be less prone to errors induced by experimental noise and speckle effects (see the variation in amplitude of fringes in Fig. 12 (a)).

For testing experimental SM signals, multiple SM signals were acquired such that PZT transducer was excited by a signal composed of a tone of $4.4 \mu \mathrm{m}$ at $170 \mathrm{~Hz}$ and another tone of $25 \mathrm{~nm}$ at $20 \mathrm{~Hz}$. SC-TFSP based motion retrieval mean of $D_{S C-T F S P}-D_{P Z T}$ based error is $1.56 \mathrm{~nm}$ with standard deviation of $0.57 \mathrm{~nm}$, that compares favorably with the reference sensor having $2 \mathrm{~nm}$ resolution (see corresponding results in Table VI. Fig. 11 and Fig. 12 demonstrate the subtraction of spectra and the recovery of tones, respectively.

\section{CONCLUSION}

The TFSP with spectral subtraction incorporates the general framework of laser phase unwrapping methodology while retaining the simple opto-electronic SM sensor design which makes it attractive for nanometric vibration measurement. Use of spectral subtraction in the TFSP significantly improves its theoretical ability to recover target vibration components 


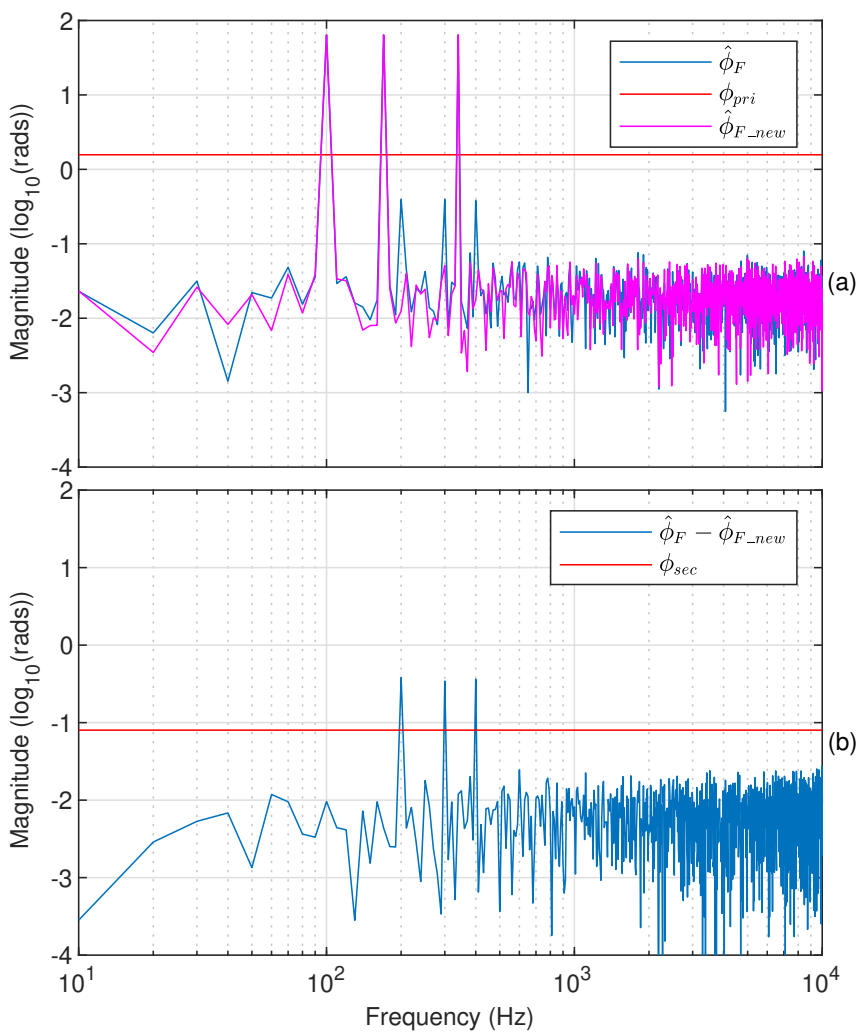

Fig. 10: Simulated result of SC-TFSP in case of target motion composed of tones of $4 \mu \mathrm{m}$ amplitude at $100 \mathrm{~Hz}, 170 \mathrm{~Hz}$ and $340 \mathrm{~Hz}$, and tones of $25 \mathrm{~nm}$ amplitude at $200 \mathrm{~Hz}, 300 \mathrm{~Hz}$ and $400 \mathrm{~Hz}$. (a) Spectrum (blue) of $\hat{\phi}_{F}$ and $\hat{\phi}_{F-n e w}$ (magenta) alongside primary threshold (red). (b) Spectrum (blue) after the subtraction alongside secondary threshold (red).

having very small amplitude (of the order of $\lambda / 150$ ) in the absence of noise. However, under experimental conditions, the proposed method could only detect remote vibrations with minimum amplitude of $\lambda / 32$ approximately. The average RMS error for experimental nanometric vibration retrieval is 1.56 $\mathrm{nm}$, with standard deviation of $0.57 \mathrm{~nm}$, for target vibrating with $25 \mathrm{~nm}$ amplitude when measured using a laser diode based SM sensor with wavelength of $785 \mathrm{~nm}$.

\section{REFERENCES}

[1] T. Taimre, M. Nikolić, K. Bertling, Y. L. Lim, T. Bosch, and A. D. Rakić, "Laser feedback interferometry: a tutorial on the self-mixing effect for coherent sensing," Advances in Optics and Photonics, vol. 7, no. 3, pp. 570-631, 2015.

[2] A. Valavanis, P. Dean, Y. L. Lim, R. Alhathlool, M. Nikolic, R. Kliese, S. P. Khanna, D. Indjin, S. J. Wilson, A. D. Rakic, et al., "Self-mixing interferometry with terahertz quantum cascade lasers," IEEE Sensors Journal, vol. 13, no. 1, pp. 37-43, 2012.

[3] A. L. Arriaga, F. Bony, and T. Bosch, "Real-time algorithm for versatile displacement sensors based on self-mixing interferometry," IEEE Sensors Journal, vol. 16, no. 1, pp. 195-202, 2016.

[4] Y. Zhao, J. Zhou, C. Wang, Y. Chen, and L. Lu, "Temperature measurement of the laser cavity based on multi-longitudinal mode laser selfmixing effect," IEEE Sensors Journal, vol. 19, no. 12, pp. 4386-4392, 2019.

[5] M. Veng, J. Perchoux, and F. Bony, "Fringe disappearance in self-mixing interferometry laser sensors: Model and application to the absolute distance measurement scheme," IEEE Sensors Journal, vol. 19, no. 14, pp. 5521-5528, 2019.
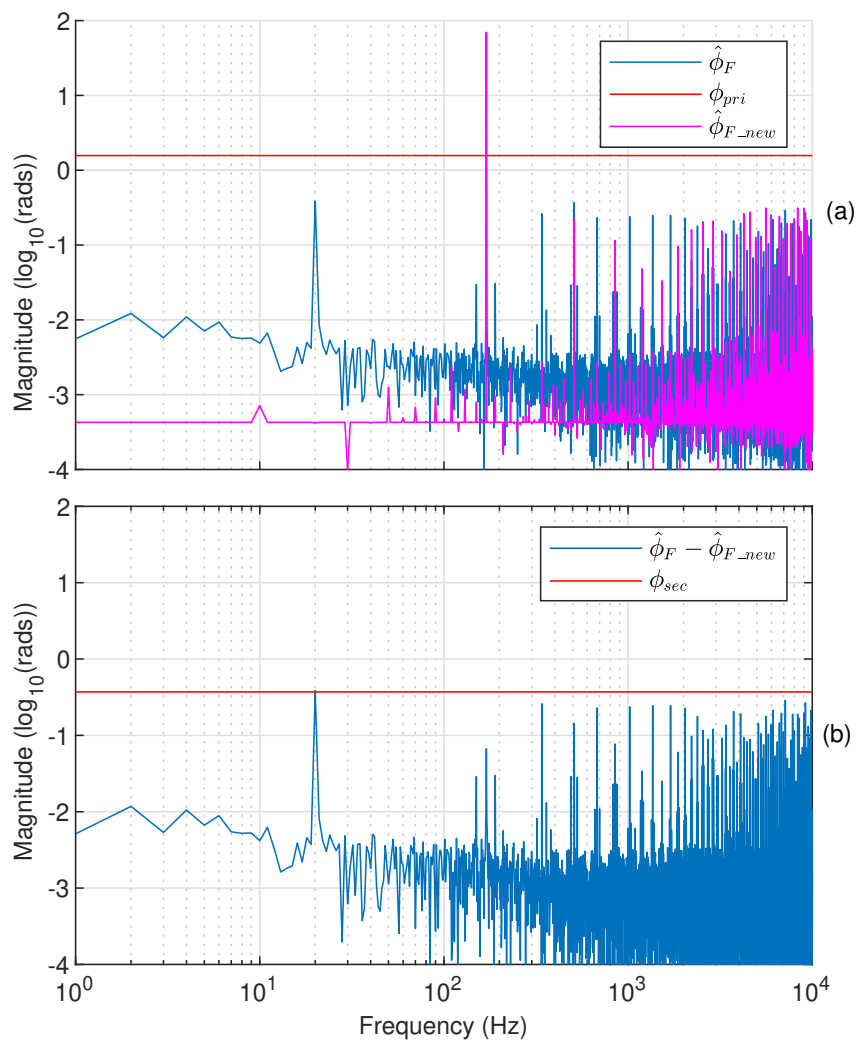

Fig. 11: Experimental result of proposed SC-TFSP in case of PZT target motion composed of a tone of $4.4 \mu \mathrm{m}$ amplitude at $170 \mathrm{~Hz}$ and another tone of $25 \mathrm{~nm}$ amplitude at $20 \mathrm{~Hz}$. (a) Spectrum (blue) of feedback phase $\hat{\phi}_{F}$ recovered from experimental SM and Spectrum (magenta) of feedback phase $\hat{\phi}_{\text {F_new }}$ of generated SM signal signal alongside primary threshold (red). (b) Spectrum (blue) after the subtraction alongside secondary threshold (red).

[6] O. D. Bernal, U. Zabit, F. Jayat, and T. Bosch, "Sub- $\lambda / 2$ displacement sensor with nanometric precision based on optical feedback interferometry used as a non-uniform event-based sampling system," IEEE Sensors Journal, vol. 20, no. 10, pp. 5195-5203, 2020.

[7] S. Ottonelli, F. De Lucia, M. Di Vietro, M. Dabbicco, G. Scamarcio, and F. P. Mezzapesa, "A compact three degrees-of-freedom motion sensor based on the laser-self-mixing effect," IEEE Photonics Technology Letters, vol. 20, no. 16, pp. 1360-1362, 2008.

[8] A. A. Siddiqui, U. Zabit, O. D. Bernal, G. Raja, and T. Bosch, "All analog processing of speckle affected self-mixing interferometric signals," IEEE Sensors Journal, vol. 17, no. 18, pp. 5892-5899, 2017.

[9] S. Merlo and S. Donati, "Reconstruction of displacement waveforms with a single-channel laser-diode feedback interferometer," IEEE Journal of Quantum Electronics, vol. 33, no. 4, pp. 527-531, 1997.

[10] C. Bes, G. Plantier, and T. Bosch, "Displacement measurements using a self-mixing laser diode under moderate feedback," IEEE Transactions on Instrumentation and Measurement, vol. 55, no. 4, pp. 1101-1105, 2006.

[11] Y. Fan, Y. Yu, J. Xi, and J. F. Chicharo, "Improving the measurement performance for a self-mixing interferometry-based displacement sensing system," Applied Optics, vol. 50, no. 26, pp. 5064-5072, 2011.

[12] O. D. Bernal, U. Zabit, and T. M. Bosch, "Robust method of stabilization of optical feedback regime by using adaptive optics for a selfmixing micro-interferometer laser displacement sensor," IEEE Journal of Selected Topics in Quantum Electronics, vol. 21, no. 4, pp. 336-343, 2014.

[13] A. Ehtesham, U. Zabit, O. D. Bernal, G. Raja, and T. Bosch, "Analysis and implementation of a direct phase unwrapping method for displacement measurement using self-mixing interferometry," IEEE Sensors 


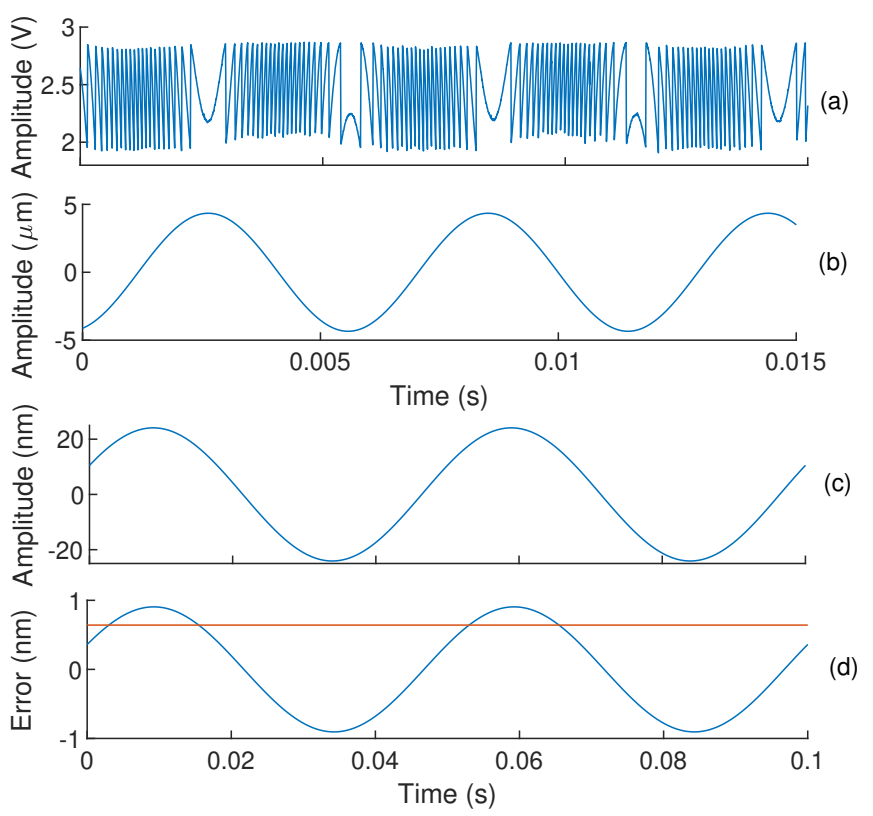

Fig. 12: (a) Experimental SM signal corresponding to PZT target vibration consisting of a main tone with $4.4 \mu \mathrm{m}$ amplitude at $170 \mathrm{~Hz}$ and a small tone with $25 \mathrm{~nm}$ amplitude at 20 $\mathrm{Hz}$, (b) recovered main tone, (c) recovered small tone, and (d) error between PZT based small tone and recovered small tone (blue), and its RMS value (red).

Journal, vol. 17, no. 22, pp. 7425-7432, 2017

[14] Z. Wei, W. Huang, J. Zhang, X. Wang, H. Zhu, T. An, and X. Yu, "Obtaining scalable fringe precision in self-mixing interference using an even-power fast algorithm," IEEE Photonics Journal, vol. 9, no. 4, pp. 1-11, 2017.

[15] C. Jiang, C. Li, S. Yin, and Z. Huang, "Multiple self-mixing interferometry algorithm based on phase modulation for vibration measurement," Optical and Quantum Electronics, vol. 49, no. 3, p. 111, 2017.

[16] Y. Zhang, R. Wang, Z. Wei, X. Wang, H. Xu, H. Sun, and W. Huang, "Broad range and high precision self-mixing interferometer based on spectral analysis with multiple reflections," IEEE Sensors Journal, vol. 19, no. 3, pp. 926-932, 2018.

[17] S. Amin, U. Zabit, O. D. Bernal, and T. Hussain, "High resolution laser self-mixing displacement sensor under large variation in optical feedback and speckle," IEEE Sensors Journal, vol. 20, no. 16, pp. 9140-9147, 2020.

[18] J. Keeley, P. Dean, A. Valavanis, K. Bertling, Y. Lim, R. Alhathlool, T. Taimre, L. Li, D. Indjin, A. Rakić, et al., "Three-dimensional terahertz imaging using swept-frequency feedback interferometry with a quantum cascade laser," Optics letters, vol. 40, no. 6, pp. 994-997, 2015.

[19] P. Dean, A. Valavanis, J. Keeley, K. Bertling, Y. Leng Lim, R. Alhathlool, S. Chowdhury, T. Taimre, L. H. Li, D. Indjin, et al., "Coherent three-dimensional terahertz imaging through self-mixing in a quantum cascade laser," Applied Physics Letters, vol. 103, no. 18, p. 181112, 2013.

[20] G. Giuliani, S. Bozzi-Pietra, and S. Donati, "Self-mixing laser diode vibrometer," Measurement Science and Technology, vol. 14, no. 1, p. 24, 2002.

[21] P. Dean, J. Keeley, A. Valavanis, K. Bertling, Y. Lim, T. Taimre, R. Alhathlool, L. Li, D. Indjin, A. Rakić, et al., "Active phase-nulling of the self-mixing phase in a terahertz frequency quantum cascade laser," Optics letters, vol. 40, no. 6, pp. 950-953, 2015.

[22] D. Melchionni, A. Magnani, A. Pesatori, and M. Norgia, "Development of a design tool for closed-loop digital vibrometer," Applied Optics, vol. 54, no. 32, pp. 9637-9643, 2015.

[23] V. Contreras, J. Lönnqvist, and J. Toivonen, "Detection of single microparticles in airflows by edge-filter enhanced self-mixing interferometry," Optics Express, vol. 24, no. 8, pp. 8886-8894, 2016.

[24] M. Norgia, D. Melchionni, and S. Donati, "Exploiting the fm-signal in a laser-diode smi by means of a mach-zehnder filter," IEEE Photonics Technology Letters, vol. 29, no. 18, pp. 1552-1555, 2017.

[25] U. Zabit, O. D. Bernal, S. Amin, M. F. Qureshi, A. H. Khawaja, and T. Bosch, "Spectral processing of self-mixing interferometric signal phase for improved vibration sensing under weak-and moderatefeedback regime," IEEE Sensors Journal, vol. 19, no. 23, pp. 11151$11158,2019$.

[26] G. Plantier, C. Bes, and T. Bosch, "Behavioral model of a self-mixing laser diode sensor," IEEE Journal of Quantum Electronics, vol. 41, no. 9 , pp. 1157-1167, 2005.

[27] O. D. Bernal, H. C. Seat, U. Zabit, F. Surre, and T. Bosch, "Robust detection of non-regular interferometric fringes from a self-mixing displacement sensor using bi-wavelet transform," IEEE Sensors Journal, vol. 16, no. 22, pp. 7903-7910, 2016.

[28] G. Giuliani, S. Donati, and W. Elsässer, "Measurement of linewidth enhancement factor of different semiconductor lasers in operating conditions," in Semiconductor Lasers and Laser Dynamics II, vol. 6184, p. 61841D, International Society for Optics and Photonics, 2006.

[29] Z. A. Khan, U. Zabit, O. D. Bernal, and T. Hussain, "Adaptive estimation and reduction of noises affecting a self-mixing interferometric laser sensor," IEEE Sensors Journal, vol. 20, no. 17, pp. 9806-9815, 2020. 